\title{
Thiolated Graphene Oxide Nanoribbons as Templates for Anchoring Gold Nanoparticles: Two-Dimensional Nanostructures for SERS
}

\author{
Jose M. González-Domínguez, ${ }^{*[a, b]}$ Andrea Colusso, ${ }^{[c]}$ Lucio Litti, ${ }^{[c]}$ Adrian Ostric, ${ }^{[a]}$ Moreno \\ Meneghetti, ${ }^{*[c]}$ Tatiana Da Ros ${ }^{\star[a]}$
}

\begin{abstract}
Graphene oxide nanoribbons (GONRs), obtained from the oxidative unzipping of carbon nanotubes, have been investigated as building blocks towards reaching active platforms in surfaceenhanced Raman scattering (SERS). The complete development of carbon nanomaterials is strongly related to the exploitation of their chemical versatility, so this work is focused on the positive effect that a specific chemical functionalization provides to the SERS effect when gold nanoparticles are used. Herein, the GONRs covalent derivatization with terminal thiol groups demonstrated to boost their interaction with gold nanoparticles, ending up in an intense enhancement of the carbon nanostructures Raman scattering itself, thanks to a two-dimensional extended aggregation pattern. Functionalized GONRs exhibit an outstanding affinity for several kinds of gold nanoparticles (namely, 'naked' or citrate-stabilized), and the two-dimensional aggregates formed thereof show SERS effect, which has been corroborated by theoretical calculations and a SERS-based sensing conceptual proof. This approach opens new venues for the use of bidimensional carbon nanostructures in SERSbased sensors.
\end{abstract}

\section{Introduction}

Since the observation of the surface-enhanced Raman scattering (SERS) phenomenon in the mid-late '70s, nowadays matching and overpassing high-sensitivity landmark techniques such as absorbance or fluorescence spectroscopies. ${ }^{[1]}$ SERS belongs to the family of molecular spectroscopies, and it is based on the plasmon-assisted scattering of molecules, which are near certain metal surfaces, supporting local surface plasmon resonance. Thus, it requires the presence of a metal, and its surface has to be nanostructured in order to achieve the highest yield, for which this technique is currently conceived mainly with metal nanoparticles. The existence of the SERS phenomenon is conditioned by the interaction of light with the plasmonic nanostructures and with the molecules located very close to the nanostructures themselves. The highest enhancements are typically observed in specific regions of the

\footnotetext{
[a] Dr. J. M. González-Domínguez, * Dr. A. Ostric, Prof. T. Da Ros * INSTM, Unit of Trieste, Department of Chemical and Pharmaceutical Sciences, University of Trieste, Via L. Giorgieri 1, $34127 \quad$ Trieste, Italy. E-mail: imgonzalez@icb.csic.es; daros@units.it

[b] Dr. J. M. González-Domínguez * Instituto de Carboquímica (CSIC), C/Miguel Luesma Castán 4 50018 Zaragoza, Spain.

[c] Dr. A. Colusso, Dr. L. Litti, Prof. M. Meneghetti *

Department of Chemical Sciences, University of Padova, Via Marzolo 1, 35131 Padova, Italy
}

E-mail: moreno.meneghetti@unipd.it nanostructures called "hot spots", where there is an electromagnetic field enhancement of orders of magnitude. Hot spots physically correspond to places near to junctions, tips and edges of nanoparticles. This is the reason why isolated spherical nanoparticles do not exhibit huge enhancements.

The deposition of a given analyte directly into the hot spot or in its very close proximity rules the overall SERS process, entailing a high spatial localization dependence. The SERS effect is especially appreciated in the bioanalytical field (direct detection of clinical analytes, whole cells, bacteria...) since it presents numerous advantages over traditional spectroscopies, namely resistance to photobleaching or photodegradation, narrow bandwidth peaks, possibility of multiplex detection and even avoidance of the intrinsic autofluorescence of the biological systems. ${ }^{[2]}$ There are examples in literature exploiting the bioanalytical potential of noble metal-based SERS systems, for example, the work reported by Yashchenok et al. ${ }^{[3]}$ These authors, designed a SERS platform supported on silica probes, based on carbon nanotubes decorated with gold nanoparticles; and this ternary system exhibited an excellent performance (even at very low laser powers), and an increased sample roughness enhancing the SERS effect. These features could be successfully employed for detecting molecular fingerprints within living cells in very benign conditions. Such results illustrate the relevance of interfacing carbon nanostructures with noble metal nanoparticles, which is able to improve the SERS response of the latter owing to their physical and chemical interactions.

The Raman features of carbon nanomaterials are rather peculiar and easy to recognize and to follow, so they can be really useful in SERS as fingerprint. For example, the use of carbon nanoparticles directly in Au hot spots provides an overwhelming contribution to the overall SERS signal, needing only few welllocated carbon nanostructures to provide a huge enhancement. ${ }^{[4]}$ Also in the past, these features have been used for such purposes, as the application of single-walled carbon nanotubes in tip-enhanced Raman scattering (TERS). ${ }^{[5]}$ This stands as a proof that carbon nanostructures exhibit great potential for Raman applications, by virtue of their unique structure-dependent properties, and the relevant optoelectronic effects taking place when interacting with noble metal nanoparticles. Likewise, the interest in SERS application of graphene, the 2-D carbon nanomaterial par excellence, is growing and the late results are promising and demonstrated its suitability, ${ }^{[6]}$ as well as for other graphene derivatives such as graphene nanoribbons (GNRs). In particular, some authors have addressed the SERS characterization of GNRs obtained by 
chemical and mechanical cleavage of pristine graphite, ${ }^{[7]}$ finding new Raman bands, due to the nanoribbon's lateral dimensions and number of layers, paving the way towards the precise characterization of width and fine edge structure in such 2-D carbon nanomaterials. Other authors have revealed a TERS enhancement factor up to $4 \cdot 10^{5}$ by directly growing armchair graphene nanoribbons on $\mathrm{Au}(111),{ }^{[8]}$ and, more recently, the combination of graphene oxide nanoribbons (GONRs) with gold has been successfully exploited to build an aptameric sensor for $\mathrm{Pb}$ (II) based on SERS principles, with high sensitivity and good selectivity. ${ }^{[9]}$ But still, deeper insights into sensitivity, selectivity and reproducibility in SERS performance are to be obtained to fully develop the potential of graphene and its related structures in such application. According to specialized literature, ${ }^{[10]}$ one of the current challenges for this to happen regards the controllable functionalization of graphene with specific chemical groups.

The contemporary use of carbon nanostructures and metal nanoparticles in SERS can be exploited both in non-specific and in targeted approaches. In the first case, this dual system can attract analytes in a not specific way, being the GONRs a platform for interactions. In a second approach, when the nanostructures are functionalized with specific targeting agents thanks to the chemical versatility of the carbon nanostructures, the SERS response will be indicative of the presence of the targeted component without any doubt, owing to the nanoribbon characteristic and the intense Raman signal. In both cases, the presence of metal nanoparticles would increase the signal by surface-enhanced Raman scattering and, if any analyte would be present, its signals would increase as well, with better performances in the detection and recognition. The use of Raman for biological purposes in presence of carbon nanostructures is already well reported, ${ }^{[11]}$ as well as the more specific use of these combinations for diagnostic approaches. ${ }^{[12]}$

In the present work, we have selected GONRs as potential candidates for SERS application. Our approach is based on the choice of a suitable surface chemistry derivatization to maximize the performance of SERS by strengthening the interactions between GONRs and gold nanostructures. More precisely, the extensive surface functionalization with very simple moieties containing terminal thiol groups has been performed as a proof of principle to induce the strongest possible binding of the carbon nanomaterial inside Au hot spots. It has been stated that the closer the metal nanoparticles are to the carbon nanostructure, the larger the SERS effect is, ${ }^{[3]}$ so a tailored chemistry between both nanostructures could play a key role in SERS. Therefore, the metal-material intensified interaction is herein intended to act as the SERS driving force, providing improved results.

To the best of our knowledge this is the first attempt on the use of chemically-functionalized GONRs in a SERS study, being the enhanced signals of the nanoribbons themselves a primary indication that the system is active in SERS. A key element is that proper chemical functionalization of the GONRs can be conceived and tailored to maximize the strength of interaction between Au hot spots and the substrate, exceedingly increasing the SERS efficiency. The interaction of analytes with graphene derivatives may also produce new states which favor the SERS enhancement with the so-called chemical mechanism in which electronic polarization or charge transfer states may help the enhancement of the SERS spectra of an analyte. ${ }^{[1]}$

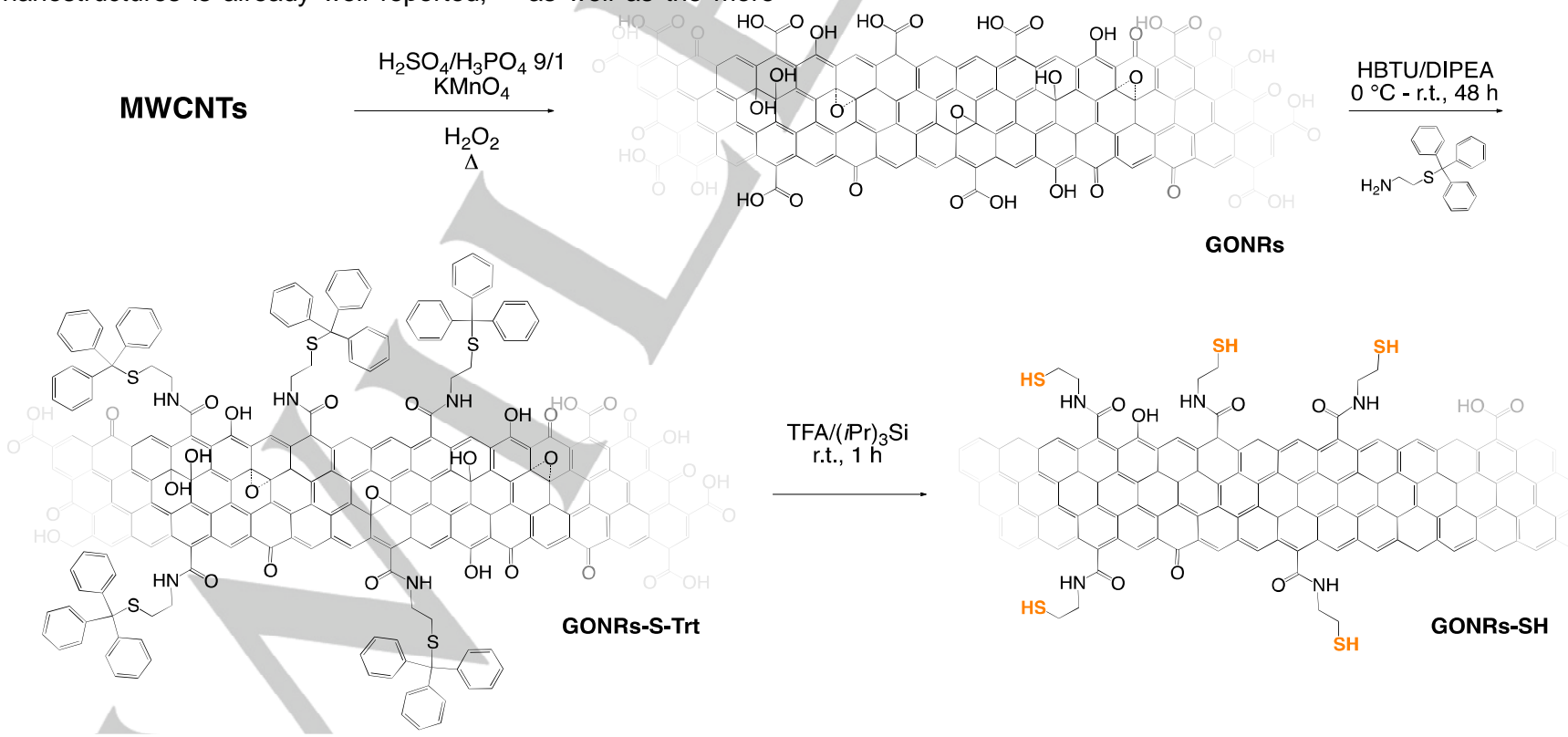

Figure 1. Preparation of thiolated GONRs. 


\section{Results and Discussion}

\section{GONRs synthesis}

The preparation of the GONRs from multi-walled carbon nanotubes (MWCNTs) was performed by treatment with acids and other strong oxidants (Fig. 1). The analysis of the successful synthesis of GONRs from MWCNTs was mainly carried out through microscopic techniques. TEM images of the pristine MWCNTs are reported in Fig. 2a-b while Figure 2c-f showed flat structures in GONRs with averaged dimensions of $480 \pm 50 \mathrm{~nm}$ in length, and $46 \pm 4 \mathrm{~nm}$ in width. While the latter is consistent with the MWCNTs diameters specified by the manufacturer, the lengths observed in GONRs suggest that MWCNTs underwent a certain degree of transversal cutting together with the unzipping mechanism. This could be associated with structural defects contained in the pristine material. ${ }^{[13]}$ The AFM analysis of GONRs and GONRs-SH (Fig. $2 \mathrm{~g}$ and $\mathrm{h}$ respectively) shows individual nanostructures with a height profile across the nanoribbons of 3-7 nm, which corresponds to the height of 2-3 graphene sheets, in agreement with typical results reported in literature. ${ }^{[14]}$ According to the MWCNT/KMnO4 ratio used in this work (1/5), and the starting MWCNTs being of a diameter higher than $15 \mathrm{~nm}$, we can conclude that the MWCNTs underwent a sequence of intercalation, oxidation and exfoliation, ${ }^{[15]}$ ending up in thin GONRs with a high level of oxidation.

\section{GONRs functionalization}

The first direct evidence of the success in GONRs functionalization was obtained by assessment of their dispersibility in different solvents (Fig. 3). As-produced GONRs were highly dispersible in water and polar solvents, such as N,N'-dimethylformamide (DMF), ethanol, and water/ethanol mixtures. However, the GONRs exhibited markedly poor dispersibility in low-polarity solvents such as dichloromethane, hexane or diethyl ether. Once the functionalization with S-Tritylmercaptoethylamine (STrt-MEA) took place, an inversion in this trend was observed, being the functionalized GONRs highly dispersible in dichloromethane and not in water. This would be in agreement with a surface that is highly covered with benzene rings, due to the Trityl capping of functional groups. Finally, the removal of Trityl groups resulted in the recovery of GONRs affinity for polar solvents. TGA characterization provided quantitative information on the extent of functionalization with MEA (Fig. 4). The starting MWCNTs presented a thermal profile in inert atmosphere with practically no weight loss in the analysis range. However, GONRs present weight losses between 100 and $300{ }^{\circ} \mathrm{C}$ ascribable to the multiple oxygen groups inserted during the oxidative unzipping process. These groups are quantified as about $40 \%$ of the total sample weight, and were taken into account for the reaction stoichiometry, stating this weight loss to be exclusively due to the presence of carboxylic acid units (see Experimental section). After functionalization with S-Trt-MEA, the measured weight loss was remarkably higher (almost $76 \%$ of weight loss at $450{ }^{\circ} \mathrm{C}$ ), corresponding to a highly functionalized material. The Trityl cleavage was performed in acid conditions and the GONR control sample helped in elucidating the effect of this treatment on the nanoribbon structure. As it has been reported ${ }^{[16]}$ graphene oxide (GO) materials are extremely versatile and reactive platforms, but their functionalization usually goes together inevitable reduction or deoxygenation. In this regard we have observed that the Trityl cleavage protocol caused such alteration to the pristine GONRs as they roughly experience just the half of weight loss in TGA experiments when subjected to TFA/silane treatment. The difference in weight loss at $450{ }^{\circ} \mathrm{C}$ between the control sample and functionalized GONR-SH materials (11\%) was used to quantitatively assess the functionalization degree. Taking into account the molecular weight of the attached moiety, we determined a content of $0.93 \mathrm{mmol}$ of thiol groups per gram of sample.
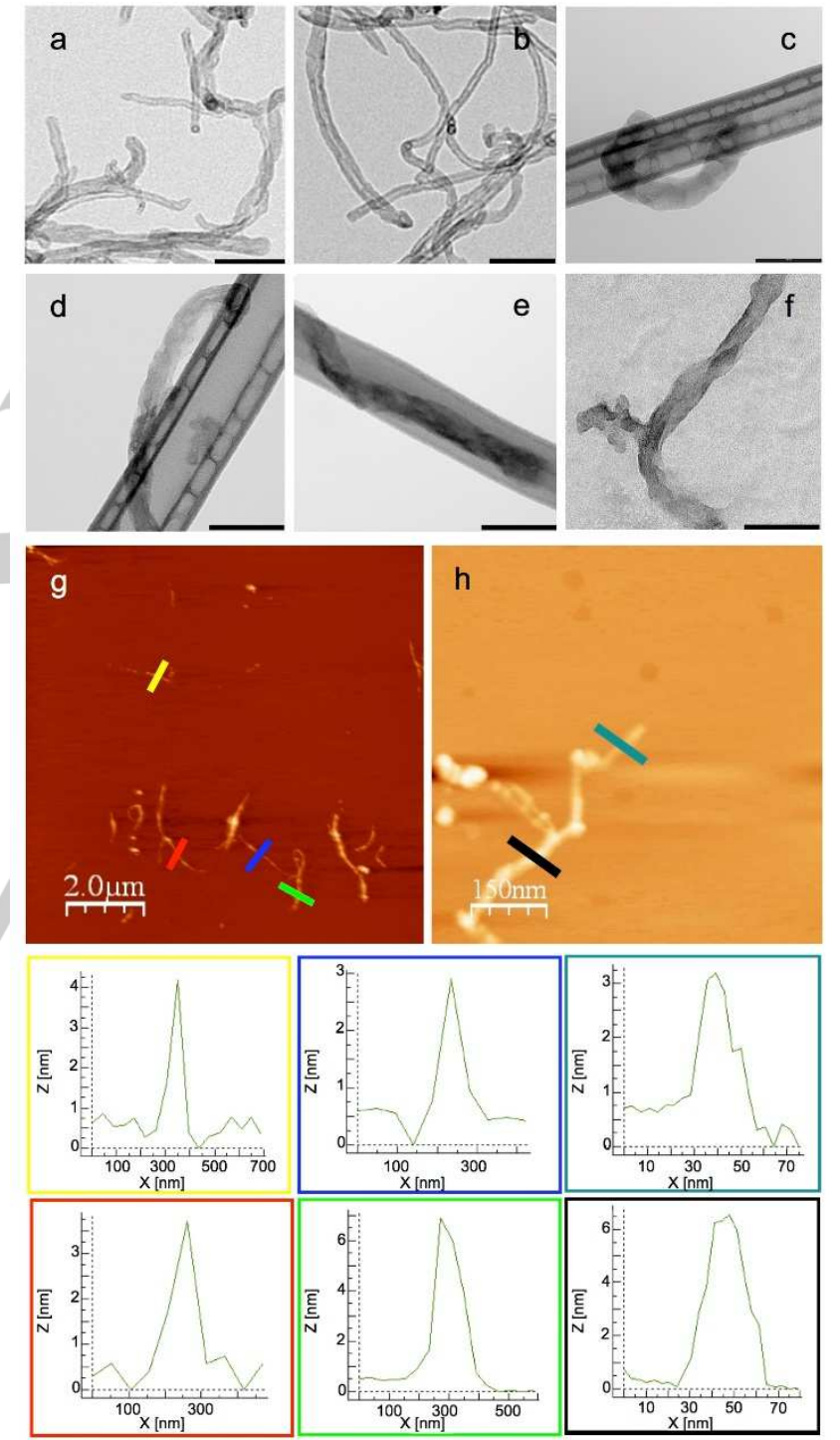

Figure 2. a-f. TEM images of commercial NanoAmor® MWCNTs $(a, b)$ and the as-synthesized GONRs (scale bars $100 \mathrm{~nm}$ ). In (c-e) the large thick bridges, over which GONRs are standing, correspond to the grid structure (Carbon lacey); carbon coated copper grid was used for f. g-h. AFM images of: g) GONRs before functionalization and h) GONRs-SH, with their corresponding height profiles (frame color corresponds to bar color). 


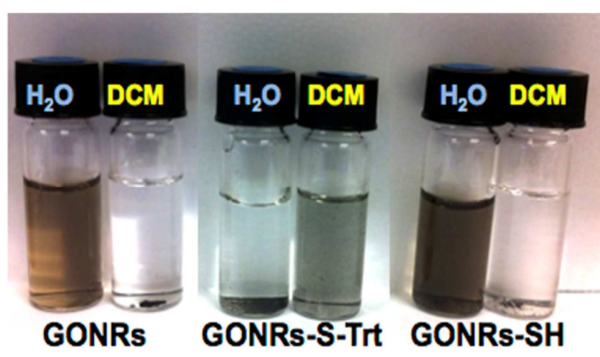

Figure 3. Dispersibility images of variously functionalized GONRs in water and dichloromethane (concentration about $0.05 \mathrm{mg} / \mathrm{mL}$ ).

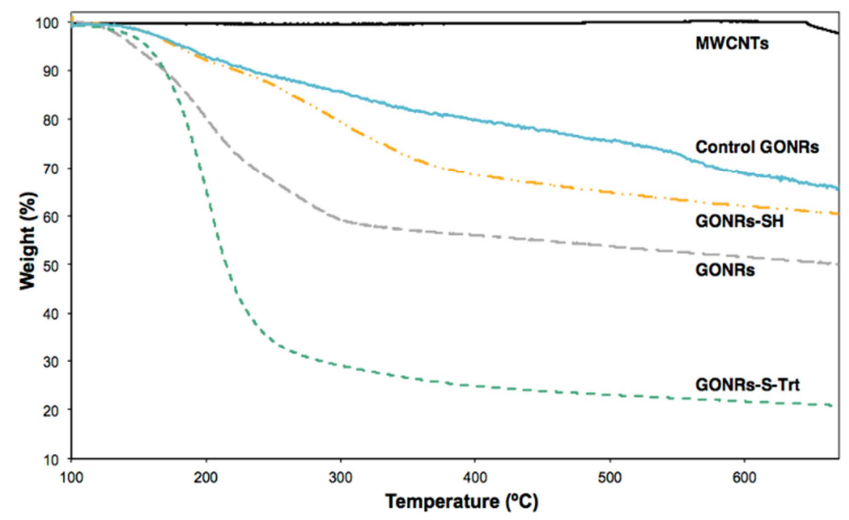

Figure 4. TGA plots of MWCNTs (solid black line), control GONRs (solid light blue line), GONRs-SH (dash-dot yellow line), GONRs (long dash grey line) and GONRs-S-Trt (short dash green line).

The presence of functional groups was confirmed by FTIR spectroscopy analyses (Fig. 5). As expected, many different oxygen groups could be distinguished in GONRs, including phenol $\left(1216 \mathrm{~cm}^{-1}\right)$, ethers and alcohols $\left(1030-1100 \mathrm{~cm}^{-1} \mathrm{C}-\mathrm{O}\right.$ stretching, $667 \mathrm{~cm}^{-1} \mathrm{O}-\mathrm{H}$ bending), oxiranes (833 and $\left.1288 \mathrm{~cm}^{-1}\right)$, carboxylate ions $\left(1590 \mathrm{~cm}^{-1}\right)$ and carboxylic acids $\left(1733 \mathrm{~cm}^{-1}\right)$. Upon functionalization with S-Trt-MEA, the most visible features were the downshift of the carboxylic band to $1680 \mathrm{~cm}^{-1}$, in agreement with the existence of amide bonds, and also the appearance of different peaks ascribable to benzene rings monosubstitution vibrations $\left(700\right.$ and $\left.744 \mathrm{~cm}^{-1}\right)$. After Trityl cleavage, benzene peaks were no longer observed.

\section{GONRs Raman profile}

In Raman spectra of MWCNTs three characteristic bands can be observed (Fig. 6): D band is at around $1350 \mathrm{~cm}^{-1}$ and it is characteristic of disordered carbon forms, thus commonly associated to structural defects; graphitic $(\mathrm{G})$ band at $1585 \mathrm{~cm}^{-1}$ which originates from in-plane vibration of $\mathrm{sp}^{2}$ carbons; and a band around $2700 \mathrm{~cm}^{-1}$ known as $2 \mathrm{D}$ band that is a result of twophonon Raman process which is independent from the presence of defects in the structure. ${ }^{[17]}$ GONRs' spectra presented much higher intensity. Raman intensity derives from many effects, one of which is the electron-vibration coupling, that also depends on the electronic structure and, so, the better delocalization of $\pi$ electrons can be beneficial in terms of signal intensity. ${ }^{[18]}$ Since electrons can delocalize better in less defective structure, we have compared ID/IG ratios of three samples (Table 1).
Surprisingly, although quite harsh process has been used on MWCNTs, the ID/IG ratio did not change considerably upon formation of GONRs. All three spectra were measured at 532 $\mathrm{nm}$ laser wavelength and they are average of 3-5 different localizations on each sample.

Table 1. Band positions and ratios drawn from Raman spectra recorded at $532 \mathrm{~nm}$..

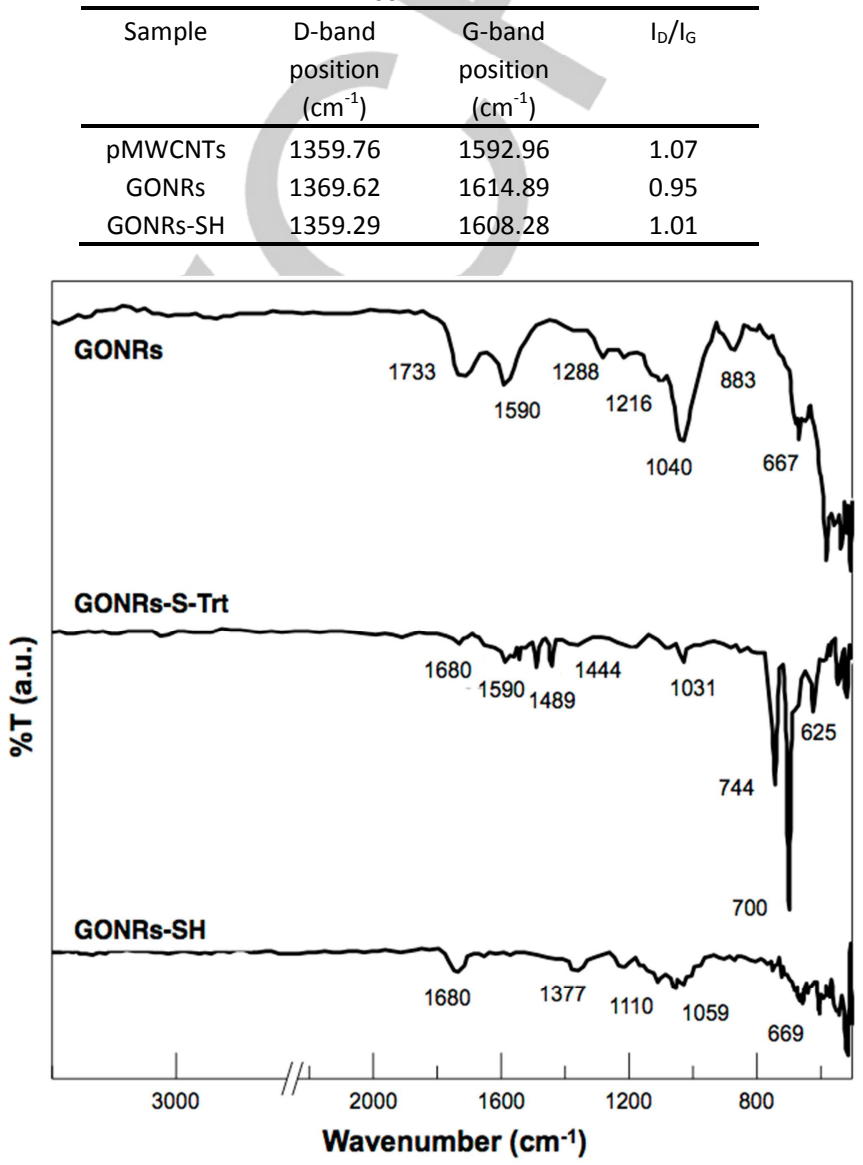

Figure 5. FTIR spectra for GONRs and their functionalized counterparts GONRs-S-Trt and GONRs-SH.

\section{SERS profile of GONRs-SH coupled to 'naked' AuNPs}

Coupling experiments between GONRs-SH and 'naked' AuNPs have been performed to assess the capability and efficacy of these systems for SERS. This coupling was obtained by mixing a solution of AuNPs at the concentration of $2 \times 10^{-9} \mathrm{M}$ with an aqueous dispersion of GONRs-SH (five times in volume) at a concentration of $0.25 \mathrm{mg} / \mathrm{mL}$. The coupling saturated all the thiol functions since, after stirring, an excess of nanoparticles was removed by centrifugation. For the Raman analysis, a smooth film of the material was obtained by dropping the solution (50 $\mu \mathrm{L}$ ) on a glass slide. Figure $7 \mathrm{a}$ shows a TEM image of a representative GONRs-S-AuNPs nanostructure in which it is possible to appreciate the AuNPs strongly coupled to the nanoribbons and arranged in 2D clusters. 
Raman spectra were recorded in ten points of the film upon excitation at $633 \mathrm{~nm}$. The average spectra (red line) are compared in Figure 7c with the spectra obtained for a film of pure GONRs-SH (black line). The difference on the films is only the presence of the AuNPs and the enhancement of the spectra recorded for GONRs-S-AuNPs is found to be about five times.

Since very small AuNPs, of the order of some nm (smallAuNPs), have lower SERS activity (see below), to confirm that the SERS enhancement observed for GONRs-S-AuNPs derived from the presence of the 2D AuNPs clusters, an analogous experiment was performed using GONRs-S-smallAuNPs. So smallAuNPs, obtained by irradiating the colloidal solution with nanosecond pulses at $532 \mathrm{~nm}$ (see Experimental section), were coupled to GONR-SH with the same protocol used for the larger ones. The colloidal solution of smallAuNPs saturated, also in this case, all the thiol functions. A representative TEM image of GONR-S smallAuNPs nanostructure is reported in Figure $7 \mathrm{~b}$, where it is possible to appreciate the presence of clusters of very small AuNPs on the surface of the nanoribbons. Raman spectrum, as the average of ten spectra recorded in different spots of a drop casted film, is reported in Figure 7c (green line): the spectrum overlaps that of pure GONR-SH, showing, as expected, almost no enhancement. This result confirms that the enhancement is a SERS enhancement derived from the AuNPs which were able to

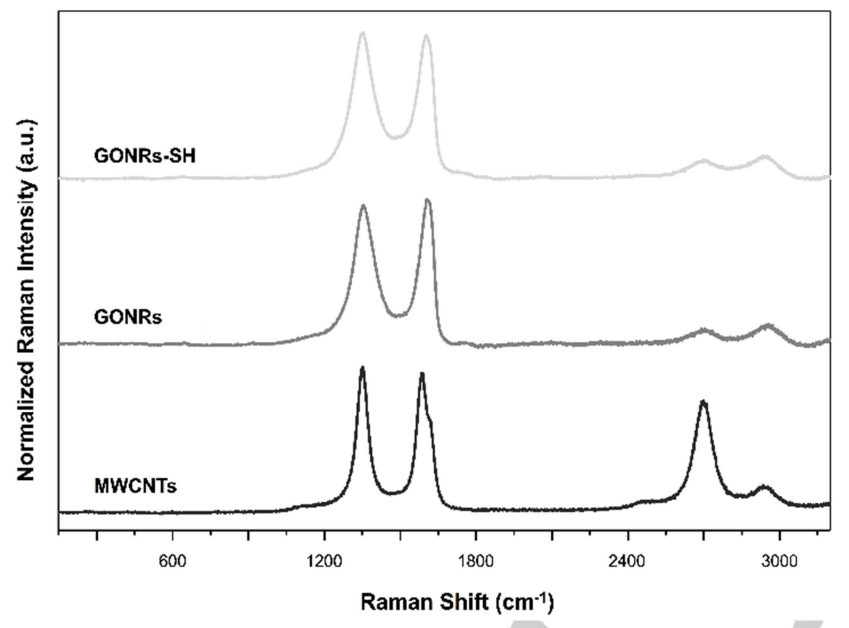

Figure 6. Raman profiles of MWCNTs (black line), GONRs (grey line) and GONRs-SH (light grey line). Excitation laser line at $532 \mathrm{~nm}$. Plots are average of at least 3-5 random localizations in each sample. Raman intensities are given in absolute value, without normalization.

produce the SERS effect.

To theoretically confirm that the smallAuNPs do not produce SERS enhancements similar to those of AuNPs, we also performed BEM (Boundary element method) ${ }^{[19]}$ calculations with six AuNPs over a graphene layer (mimicking the GONRs) with diameters randomly varied between 15 and $25 \mathrm{~nm}$ (AuNPs, Fig. $8 \mathrm{a})$ and between 1 and $3 \mathrm{~nm}$ (smallAuNPs, Fig. 8b). The simulated geometries take inspiration directly from the TEM images of Figure $7 \mathrm{a}$ and $7 \mathrm{~b}$, fixing a minimum distance between the particles and the graphene layer to $0.5 \mathrm{~nm}$. The maximum enhancement calculated within the hot spots by exciting at 633 $\mathrm{nm}$ was found to be of $4 \cdot 10^{7}$ for the AuNPs and about three orders of magnitude less for the case of the smallAuNP, so the largest SERS effects were found, as expected, between bigger AuNPs. Very small enhancements, of the order of $10^{2}$ and similar to those of isolated nanoparticles, were also calculated between AuNPs and the graphene layer. This showed that using particles with larger diameters it is possible to obtain much larger SERS enhancements. Stronger enhancements were calculated for the GONRs-S-AuNPs also using different excitation wavelengths.

The experimental enhancements, however, seem to be much smaller than the calculated ones. It is necessary to highlight that the experimental Raman spectrum is an average spectrum of the GONRs in the sample, but that only a very small amount of nanoribbons are probably present within the hot spots, where the enhancement takes place. Therefore, the absolute values of the real enhancements are much larger but difficult to record experimentally and only a smaller and apparent value enhancement can be observed.

The enhancement of the graphene Raman signals with the presence of the AuNPs (see Table 2) shows that the nanostructured system is an active material for SERS and this can be exploited for any analyte that can be captured by the GONRs, either in a specific way (if targeting agents are coupled to the carbon nanostructures) or in a non-specific manner, as previously mentioned.

\begin{tabular}{|c|c|c|c|c|}
\hline \multirow{4}{*}{$\begin{array}{l}\text { Table } 2 \text {. } \\
\text { Intensity } \\
\text { (CPS) of } D \\
\text { and G bands } \\
\text { and ratios } \\
\text { from Raman }\end{array}$} & & & & \\
\hline & Sample & $1352 \mathrm{~cm}^{-1}$ & $1579 \mathrm{~cm}^{-1}$ & $\mathrm{I}_{\mathrm{D}} / \mathrm{I}_{\mathrm{G}}$ \\
\hline & GONRs-SH & 27 & 28 & 0.96 \\
\hline & $\begin{array}{l}\text { GONRs-S- } \\
\text { mallAuNPs }\end{array}$ & 29 & 27 & 1.07 \\
\hline & $\begin{array}{l}\text { GONRs-S- } \\
\text { AuNPs }\end{array}$ & 117 & 124 & 0.94 \\
\hline
\end{tabular}

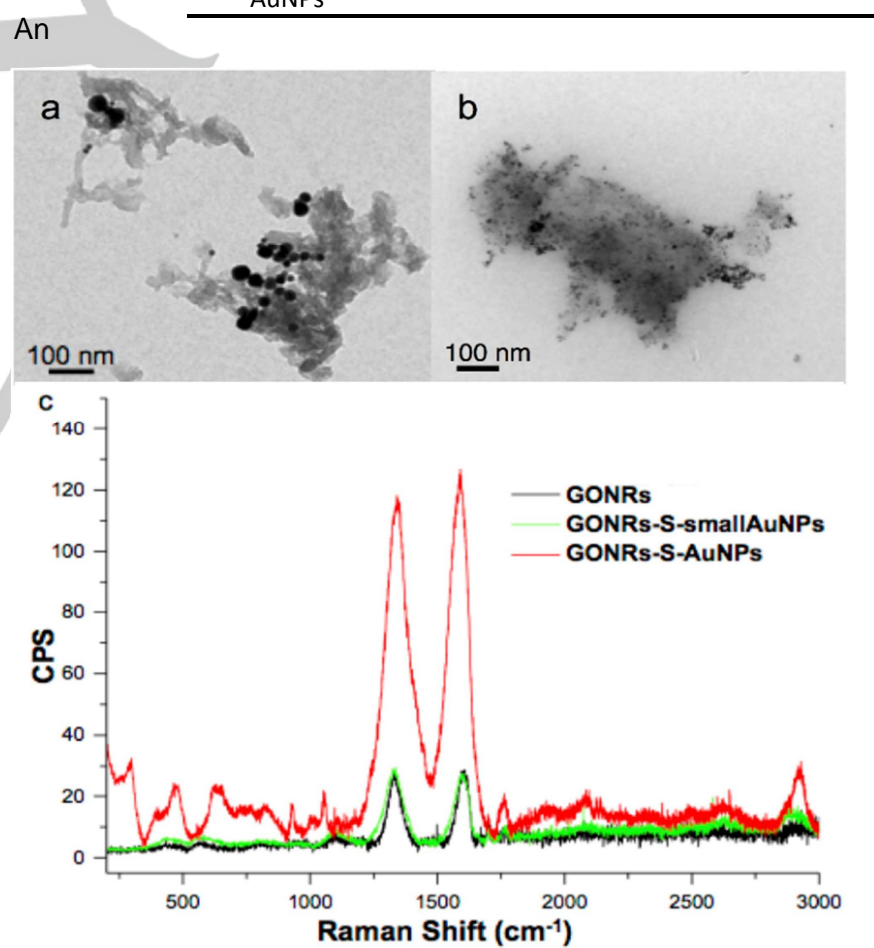

Figure 7. a) TEM image of GONRs-S-AuNPs; b) TEM image of GONRs-SsmallAuNPs on carbon coated copper grids; c) Raman spectra of films of GONRs-SH (black line), GONRs-S-AuNPs (red line) and GONRs-SsmallAuNPs (green line). Spectra are the average of spectra recorded on ten points of the film. Excitation laser line at $633 \mathrm{~nm}$.

additional feature observed from the enhanced Raman signals in Figure 7 (red spectrum) is the emergence of previously unseen bands in the spectral profile. Since there is no external analyte in 


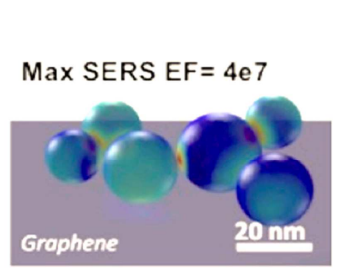

(a)

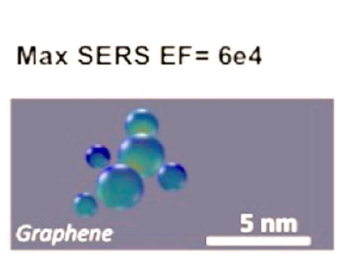

(b)

Figure 8. BEM calculations of the SERS enhancement factor for cluster of six AuNPs of dimension in the range a) $15-25 \mathrm{~nm}$ and b), 1-3 nm, randomly aggregated, over a graphene layer. The scale bar reports the values for the enhancement factors. Hot spots can be observed between nanoparticles (red spots)

these measurements, the new bands could be ascribed to the GONRs-SH functional groups, which arise from the intensity enhancement. According to fundamental studies on the Au-S interfacial chemistry, an evidence of the thiol binding to gold is demonstrated through specific Raman bands corresponding to $\mathrm{S}^{2-}$ species at $200-350 \mathrm{~cm}^{-1}$ and also around $500 \mathrm{~cm}^{-1}$. ${ }^{[20]}$ This confirms not only the existence of thiol groups in GONRs-SH but also that the driving force causing the SERS effect is a selfassembled aggregation between thiol groups and AuNPs, ruled by the basic principles of this kind of chemistry.

The enhancement of the graphene Raman signals with the presence of the AuNPs (see Table 2) shows that the nanostructured system is an active material for SERS and this can be exploited for any analyte that can be captured by the GONRs, either in a specific way (if targeting agents are coupled to the carbon nanostructures) or in a non-specific manner, as previously mentioned. Similar situations were observed for example with the intercalation of $\mathrm{WS}_{2}$ between AuNPs. ${ }^{[21]}$
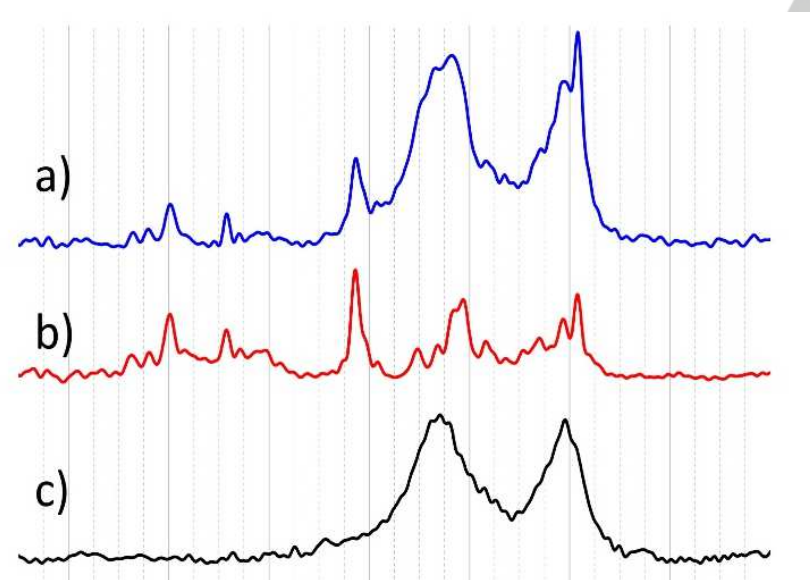

$\begin{array}{llllllllll}600 & 800 & 1000 & 1200 & 1400 & 1600 & 1800 & 2000\end{array}$

Raman Shift $\left(\mathrm{cm}^{-1}\right)$

Fgure 9. SERS spectra (633 nm laser) of: a) GONRs-S-AuNPs substrate after its immersion in a $1.3 \mu \mathrm{M}$ Crystal Violet solution; b) reference SERS spectrum of a Crystal Violet; and c) the SERS spectrum of GONRs-S-AuNPs substrate before the immersion in the Crystal Violet solution.

In order to show that the nanostructures are an active SERS substrate the GONRs-S-AuNP, deposited on a glass slide, were immersed (1 second) in a $1.3 \mu \mathrm{M}$ solution of Crystal Violet
(Sigma Aldrich \#HT901), a molecule used as SERS reporter. Figure 9 shows the recorded spectrum, after drying the substrate, and, for comparison, those of GONRs-S-AuNP and of the SERS spectrum of Crystal Violet. As one can clearly see the recorded spectrum shows both the enhanced signals of GONRs and of Crystal Violet. In particular, there is one band from Crystal Violet that outstands in the enhanced spectrum, namely the one at $1600 \mathrm{~cm}^{-1}$, as a consequence of the SERS effect from the GONR-S-AuNPs constructs.

\section{GONR-SH interaction with citrate-stabilized Au nanoparticles}

In order to further prove the validity for SERS of the constructed structures we also used capped AuNPs for the functionalization of the GONRs-SH. It is known from many studies that AuNPs capped with non-sulfur stabilizing species have indeed higher binding affinity to sulfur than for the own stabilizers. In particular, citrate-stabilized AuNPs exhibit acomplex Au-S interface, with a full removal of citrate anions if exposed to sulfide aqueous solutions. The treated AuNPs do not show any sign of oxidation in such case and the nucleophilic interaction of the sulfurcontaining species appear (as evidenced by spectroscopic techniques) as a mixture of sulfides, polysulfides and elemental sulphur. ${ }^{[20]}$ For this reason, the SERS potential of the $2 \mathrm{D}$ platforms described herein would be fully validated if they show strong binding affinity also for capped AuNPs.
In order to prove this premise, the AuNPs water colloid, stabilized with citrate anions (cAuNPs, mean diameter $=15.3 \mathrm{~nm}$, $\left.\mathrm{c}=4.22 \times 10^{-9} \mathrm{M}, \mathrm{c}=0.1 \mathrm{mg} / \mathrm{mL}\right),{ }^{[22]}$ was mixed (1:1 in volume) with an aqueous dispersion of GONRs or GONRs-SH with a concentration of about $0.05 \mathrm{mg} / \mathrm{mL}$, in a glass vial and handshaked for five minutes. The typical reddish colour of the cAuNPs immediately disappeared when mixed with GONRs-SH, while no appreciable changes were observed to the mixture containing as-prepared GONRs and cAuNPs (insets in Fig. 10a). After a short time of settlement at room temperature, the UV-Vis spectra were recorded and it was clear that a strong interaction occurred between GONRs-SH and cAuNPs as a new band with maximum at around $670 \mathrm{~nm}$ appeared, corresponding to the new plasmonic transition attributable to the gold clusters formed on the GONRs-SH surface. These results are analogous to that obtained for naked AuNPs, with similar UV-Vis spectroscopic profiles as observed for cAuNPs (data not shown).

The high affinity of GONRs-SH towards cAuNPs was observed with TEM (Figure 10b). The microscopic visualization of these mixtures revealed that GONRs-SH form robust aggregates and act as bridges amongst many particles. This affinity-induced aggregation of cAuNPs was not observed in plain GONRs, where these nanoparticles appeared randomly scattered across the TEM grid surface and almost no interaction with GONRs was denoted, with the presence of only few cAuNPs mostly located at the GONRs edges (data not shown). 
So the affinity of thiols for cAuNPs appears to be consistent as underlined also by the presence of the broad band at about 670 $\mathrm{nm}$ in the UV-Vis spectrum. As mentioned, it is related to plasmon resonances of cAuNPs clusters, confirming the aggregation on the GONRs-SH, and demonstrating that the synthesized GONRs-SH can be functional not only in presence of naked AuNPs but also with citrate stabilized AuNPs (cAuNPs). In some literature works, AuNPs aggregation is controlled by addition of ionic compounds such as $\mathrm{NaCl}$, hinted by a colour change of the solution, from red to blue. ${ }^{[23]}$ By cross-comparing
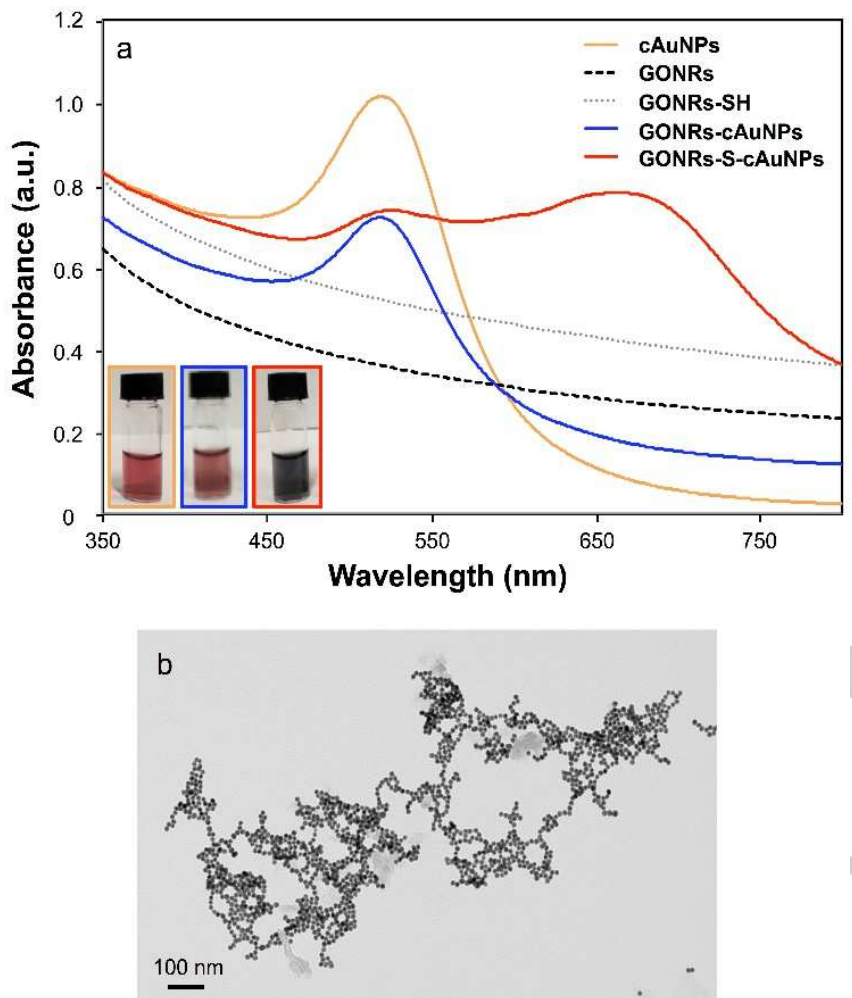

this classical way of aggregation with the one presented herein through the gold-thiol chemistry at the interface with the nanocarbon substrate, the choice of optimal conditions does not rely in a specific color change, but rather in a disappearance of the red color (in favor of the typical black background of carbon nanoforms) which can be tracked by naked eye with high precision. The salt-induced AuNPs aggregation can be circumvented if the substrate is provided with a suitable interfacial chemistry.

Figure 10. a) UV-Vis spectra for cAuNPs (light grey solid line), GONRs (dashed line), GONRs-SH (dotted line), GONRs-cAuNPs (black line) and GONRs-S-cAuNPs (grey line); the insets show the water solution of cAuNPs (light grey frame), GONRs-cAuNPs (black frame) and GONRs-S-cAuNPs (grey frame); b) TEM images for GONRs-S-cAuNPs.

\section{GONRs versus other carbon nanostructure in SERS applications}

We demonstrated that GONRs are ideal candidates as substrate in SERS. The key issue was the exploitation of their vast chemical versatility to incorporate thiol groups, thus creating a strong chemical interface with AuNPs. This can be considered an advantage when compared to non-oxidized similar nanostructures as GNRs. However, this versatility is a feature common also to other similar carbon nanostructures (i.e. seamless carbon nanotubes (CNTs) or traditional graphene oxide (GO)). However, these latter materials present respectively narrow width and high aspect ratio (CNTs) or bidimensional character and rich surface chemistry (GO), while GONRs gather both characteristics, which are two of the most useful features for good interactions with a number of AuNPs, allowing GONRs to outperform with respect to other materials. These joint features are the key for the successful results obtained, since the small lateral dimensions coupled to the tailored surface chemistry has led to an efficient bidimensional aggregation, which maximizes the number of hot spots. Similar works performed with functionalized ${ }^{[24]}$ or non-functionalized CNTs, ${ }^{[25]}$ and also with GO, ${ }^{[26]}$ showed sidewall or basal plane decoration with AuNPs but seemingly not in extended 2D aggregates with many hot spots. In this regard, some examples have been reported on SERS sensors based on such AuNPdecorated carbon nanostructures, namely recyclable SERS membranes made of intercalated graphene oxide, ${ }^{[27]}$ or nanoprobes for intracellular single-cell detection. ${ }^{[28]}$

We postulate that our strategy is an optimal way to obtain both the strongest Au-substrate interactions and the maximum possible number of hot spots. The present approach also shows to be very efficient to obtain SERS spectra of GONRs, which can be useful for tracking these nanostructures in different environments, like the biological one, but also offers the possibility of obtaining hot spots for the usual applications of SERS such as the determination of analytes. In the last cases GONRs can be also centers for selective interactions with analytes.

\section{Conclusions}

In summary, considering the great interest on carbon nanomaterials, we explored how GONRs can be used for SERS enhancement. GONRs were prepared by oxidation and unzipping process of MWCNTs. This methodology also allowed to decrease the length of the pristine structures, obtaining bidimensional flakes of about $480 \times 46 \mathrm{~nm}$. The chemical functionalization of these GONRs with terminal thiol groups promoted a very strong interaction between GONRs and AuNPs, together with the formation of 2D AuNPs clusters, leading to a positive effect for SERS. The presence of thiol pending groups resulted to be very important for linking and organizing the AuNPs on the surface of GONRs in such bidimensional arrangement, paving the way towards innovative constructs with potential to become useful SERS platforms. The SERS enhancement of the graphene Raman signals shows that the constructed nanostructures can be used as active SERS substrates.

\section{Experimental Section}

\section{Materials and Reagents}

MWCNTs were purchased from Nanostructured \& Amorphous Materials, Inc.® commercial source. This material (Stock \# 1229 YJ; Lot. \# 1229 
071610) was received as a mixture of MWCNTs with different number of concentric layers and average dimensions of 10-30 $\mu \mathrm{m}$ in length and 20$30 \mathrm{~nm}$ in diameter. All the other reagents were acquired from Carlo Erba (Phosphoric acid, trifluoroacetic acid), Fluka (Potassium Permanganate, Mercaptoethylamine), Alfa Aesar (organic solvents, HBTU) and Sigma Aldrich (Hydrochloric acid, Sulphuric acid, Hydrogen peroxide $30 \%$, DIPEA, Triisopropyl silane, Trityl chloride, $\mathrm{NaOH}$ ) in synthesis grade and used without further treatments.

\section{Synthetic procedures}

Preparation of S-Trityl (S-Trt) mercaptoethylamine (MEA). MEA (1.5 $\mathrm{g}, 14.4 \mathrm{mmol}$ ) and trifluoroacetic acid $(2.22 \mathrm{~mL}, 28.7 \mathrm{mmol}$ ) were dissolved in $20 \mathrm{~mL}$ of dry dichloromethane (DCM) under argon atmosphere. Upon addition of $4.2 \mathrm{~g}(15.1 \mathrm{mmol})$ of Trityl chloride, the solution turned dark yellow. After $30 \mathrm{~min}$ of stirring at room temperature under argon atmosphere, the reaction was quenched with $30 \mathrm{~mL}$ solution of $\mathrm{NaOH} 1 \mathrm{M}$. The organic layer was diluted with $75 \mathrm{~mL}$ of fresh $\mathrm{DCM}$ and additional $20 \mathrm{~mL}$ of $\mathrm{NaOH} 1 \mathrm{M}$ solution were added. The organic laye was recovered, washed with Brine solution and dried over anhydrous NaSO4. The evaporation of DCM gave $4.8 \mathrm{~g}$ of yellow oil, which was purified by flash chromatography on silica gel using EtOAc/MeOH (4/1) as eluent. After trituration with diethyl ether $3.5 \mathrm{~g}(76 \%)$ of white solid were obtained. $1 \mathrm{H} \mathrm{NMR} \mathrm{(500} \mathrm{MHz,} \mathrm{CDCl3):} \delta=7.27-7.03(\mathrm{~m}, 15 \mathrm{H})$, 2.54 (br, 2 H) 2.32 (bt, 2 H), 2.20 (bt, 2 H). Data in accordance with literature. ${ }^{29]}$

GONRs production. The top-down approach of longitudinal unzipping of MWCNTs with strong oxidants in acid medium was used. ${ }^{[14]}$ Besides, an improved method based on the addition of a second acid $^{[30]}$ was followed in order to obtain GONRs with higher quality, lower defects and sharpened aspect ratios. In a typical experiment, $150 \mathrm{mg}$ of MWCNTs were placed in a round-bottom flask, soaked in $150 \mathrm{~mL}$ of a sulfuric/phosphoric (90/10) acid mixture and bath-sonicated (Cleanosonic $® 40 \mathrm{KHz}$ ) for $1 \mathrm{~h}$. Then, the suspended mixture was heated to $65{ }^{\circ} \mathrm{C}$ and magnetically stirred for another $1 \mathrm{~h}$. After this time, $750 \mathrm{mg}$ of $\mathrm{KMnO}_{4}$ were incorporated, and the mixture was allowed to react for $2 \mathrm{~h}$ at $65^{\circ} \mathrm{C}$. The reaction medium was cooled down to room temperature for about $30 \mathrm{~min}$ and poured onto $400 \mathrm{~g}$ of ice containing also $10 \mathrm{~mL}$ of $\mathrm{H}_{2} \mathrm{O}_{2}$. The cold blend was left to settle down overnight and afterwards filtrated through a PTFE membrane $(0.1 \mu \mathrm{m}$ pore size). The dark solid collected over the membrane was re-dispersed, assisted by ultrasounds bath, in $150 \mathrm{~mL}$ of deionized water and then $15 \mathrm{~mL}$ of concentrated commercial $\mathrm{HCl}$ were added. The blend was again left to settle down overnight and filtered in identical conditions. The collected solid was re-dispersed again in $150 \mathrm{~mL}$ of absolute ethanol and to this fraction $150 \mathrm{~mL}$ of an ethyl ether/hexane mixture (1/1) were stepwise added until a solid precipitate was observed. Finally, the solvents were decanted and the GONRs were collected by filtration, obtaining a quantitative yield in weight. From the initial $150 \mathrm{mg}$ of MWCNTs, we eventually obtained $225 \mathrm{mg}$ of GONRs, which means a $150 \%$ mass yield production of the latter. This is in perfect agreement with the highly oxidative conditions employed during the synthesis.

GONRs functionalization with MEA. From the TGA characterization, we observed that the GONRs presented a weight loss of around $40 \%$ in the range of $100-300{ }^{\circ} \mathrm{C}$, which was used as an (over)estimation of the carboxylic acids quantity present on GONRs, attributing the entire weight loss to this functional group. Thus, the so prepared GONRs presented approximately $9 \mathrm{mmol}$ of $\mathrm{COOH}$ per gram of sample. Taking this into account, the functionalization was performed as follows. $20 \mathrm{mg}$ of GONRs were dispersed in about $20 \mathrm{~mL}$ of deionized water with the aid of ultrasound bath. After reaching a stable dispersion state, the liquid medium was cooled down to $0^{\circ} \mathrm{C}$ with an ice bath. Then, $60 \mathrm{mg}(0.16$ $\mathrm{mmol}$, 1.2 eq. with respect to overestimated amount of $\mathrm{COOHs}$ ) of O(Benzotriazol-1-yl)-N,N,N , ,N ' -tetramethyluronium hexafluorophosphate (HBTU) and $400 \mu \mathrm{L}$ of N,N-diisopropylethylamine (DIPEA) were added and the mixture was stirred at $0^{\circ} \mathrm{C}$ for $1 \mathrm{~h}$ under argon atmosphere. Afterwards, $57.7 \mathrm{mg}(0.18 \mathrm{mmol})$ of S-Trityl MEA were incorporated and the reaction was allowed to undergo at room temperature for $48 \mathrm{~h}$ under argon atmosphere. After this time, the sample was placed in a pre-hydrated dialysis sack (Sigma Aldrich D6066, 12kDa cutoff) and dialyzed with deionized water. Finally, the sample was lyophilized and $45 \mathrm{mg}$ were recovered. The cleavage of Trityl group was performed in acid medium. $20 \mathrm{mg}$ of functionalized GONRs were bathsonicated in $25 \mathrm{~mL}$ of DCM and then $200 \mu \mathrm{L}$ of trifluoroacetic acid and 60 $\mu \mathrm{L}$ of triisopropylsilane (used as scavenger to avoid reversion of Trityl cleavage $)^{[31]}$ were added. The mixture was left stirring at room temperature for $90 \mathrm{~min}$. The reaction medium was eventually filtrated through a PTFE membrane $(0.45 \mu \mathrm{m}$ pore size), copiously washed with DCM and diethyl ether and dried and $7 \mathrm{mg}$ were recovered. A control sample was prepared for comparative purposes and it consisted basically in an aliquot of GONRs directly subjected to the same protocol of Trityl cleavage without undergoing the previous reaction with MEA. In this case $75 \%$ of yield in weight was obtained.

Gold nanoparticles preparation. Gold nanoparticles (AuNPs) were obtained through a physical approach, namely LASiS (Laser Ablation Synthesis in Solvents). LASiS is a "green" technique for the synthesis of stable noble metal nanoparticles in water or in organic solvents, without stabilizing molecules. ${ }^{[27]}$ Briefly, the synthesis is obtained by focusing 6 ns pulses at $1064 \mathrm{~nm}$ of a Nd:YAG laser (Quantel Brilliant 50) on a target of pure gold (99.999\%) under a $10^{-5} \mathrm{M} \mathrm{NaCl}$ water solution. AuNPs obtained by LASiS show a mean diameter of $20 \mathrm{~nm}$. The stability of the colloidal solution derives from the surface charges that the particles show after the laser ablation synthesis. ${ }^{[32]}$ The "naked" nanoparticles have a free surface, allowing an easy functionalization simply by mixing the nanoparticles colloidal solution with the nanoribbon dispersion. Smaller AuNPs (smallAuNPs) are obtained by irradiation of the colloidal solution with Tween ${ }^{\circledR} 20$ at a concentration of $0.5 \%$ (in volume), using the duplicated Nd:YAG laser pulses at $532 \mathrm{~nm}$ for 40 minutes. By slow centrifugation, sAuNPs with diameters in the range $2-5 \mathrm{~nm}$ are obtained. The citrate-coated gold nanoparticles (cAuNPs) have been prepared as already described. ${ }^{[22 b]}$

\section{Characterization techniques}

Thermogravimentric analysis (TGA) was recorded with a TGA Q500 (TA instruments), under a flow of $\mathrm{N}_{2}(90 \mathrm{~mL} / \mathrm{min})$, following a temperature program consisting of the equilibration of the sample at $100{ }^{\circ} \mathrm{C}$, and followed by a ramp at $10^{\circ} \mathrm{C} / \mathrm{min}$ up to $800^{\circ} \mathrm{C}$. The sample aliquot ranged from 1 to $2 \mathrm{mg}$, exactly weighed.

Raman spectra were recorded by exciting at $633 \mathrm{~nm}$ and using a 20x objective of a Leica microscope coupled with an inVia Renishaw microRaman instrument (power on the sample was below $0.3 \mathrm{~mW}$ ) or using a 50x objective and a laser source at $532 \mathrm{~nm}$ with the same instrument. In the latter case, the laser power was maintained at $0.5 \%$ of the maximum power, which was below $0.9 \mathrm{~mW}$, in order to avoid burning or damaging the sample. ${ }^{[33]}$ The presented spectra are an average of at least 3-4 nearby points. The UV-Vis-NIR spectroscopy measurements were carried out with a Cary 5000 spectrophotometer by using $10 \mathrm{~mm}$ path length quartz cuvettes.

TEM measurements were performed with a Philips EM208 device by using an accelerating voltage of $100 \mathrm{kV}$. The samples were prepared by drop casting an ethanol/water (50/50) dispersion or the dispersion of interest directly onto a TEM grid. The grids were 300 mesh carbon- 
coated copper grid or carbon-coated lacey copper grids (as mentioned in the captions to figures).

Infrared analysis was performed by using a Fourier-transform infrared (FTIR) spectrometer (Variant 660-IR) with Pike Gladi ATR module. A small portion of the solid samples was directly measured in the attenuated total reflectance (ATR) mode and pressed in a CdSe crystal. The spectra were collected after 64 accumulations and then baselinetreated and smoothed with Origin 8.0 software tool.

AFM measurements were performed with a Multimode SPM from Veeco Instruments (Santa Barbara, US), equipped with Nanoscope V controller and JV-scanner (130 $\mu \mathrm{m}$ scan size in $\mathrm{XY}$, and $6 \mu \mathrm{m}$ Z-range), and placed on a passive, air-damped vibration isolation table from Technical Manufacturing Company (Boston, US). Silicon phosphide tips were used, and samples were registered in tapping mode.

\section{Boundary Element Method (BEM) calculations}

The local fields simulations were done using the MNPBEM libraries developed by Hohenester et al. ${ }^{[19]}$ The excitation fields are considered as propagating along the $\mathrm{x}, \mathrm{y}$ and $\mathrm{z}$ axes and polarized along the two other normal directions. The results are an average of the different field excitations, simulating the situation of a nanostructure at a random orientation with respect of the incoming field.

BEM simulations ${ }^{[19 b]}$ were produced with structures inspired by the TEM images reported in Figure $8 \mathrm{a}$ and $8 \mathrm{~b}$. A graphene layer (refractive index from http://refractiveindex.info) of $200 \times 300 \mathrm{~nm}$ large and $5 \mathrm{~nm}$ thick was used. Random dimensions were chosen for AuNPs and smallAuNPs and the spheres were produced with 400 verts polyhedra. The assemblies were built by random positioning the spheres over the graphene layer fixing a minimum distance of $0.5 \mathrm{~nm}$ between each of them. This is considered to be the minimum distance to avoid complex coupling effects. $^{[34]}$ The excitation field (at $633 \mathrm{~nm}$ ) was considered as propagating along the $x, y$ and $z$ axes and polarized along the two other normal directions. The results are an average of the different field excitations, simulating the situation of a nanostructure at a random orientation with respect to the incoming field.

\section{Acknowledgements}

$A C, L L$ and MM would like to thank the University of Padova for funding (P-DiSC \#04BIRD2016-UNIPD and the strategic program NAMECA). This project has received funding from the European Union's Horizon 2020 Research and Innovation Programme under the Marie Skłodowska-Curie Grant Agreement $n^{\circ} 734834$ (INFUSION) and from the PEOPLE Programme (Marie Curie Actions) of the European Union's Seventh Framework Programme FP7 Grant Agreement $n^{\circ}$ 290023 (RADDEL).

Keywords: graphene $\cdot$ gold $\bullet$ nanoparticles $\cdot$ SERS $\bullet$ thiols

[1] S. Schlücker, Angew. Chem. Int. Ed. 2014, 53, 4756-4795.

[2] a) C. Zhong, M. Xu, L.-J. Xu, T. Wei, X. Ma, X.-S. Zheng, H. Ren, B. Ren, Chem. Rev., 2018, 118, 4946-4980; b) M. Meneghetti, A. Scarsi, L. Litti, G. Marcolongo, V. Amendola, M. Gobbo, M. Di Chio, A. Boscaini, G. Fracasso, M. Colombatti, Small 2012, 8, 3733-3738
[3] A.Yashchenok, A. Masic, D. Gorin, B. S. Shim, N. A. Kotov, P. Fratzl, H. Möhwald, A. Skirtach. Small 2013, 9, 351-356.

[4] C. Chen, J.A. Hutchison, F. Clemente, R. Kox, H. Uji-I, J Hofkens, L. Lagae, G. Maes, G. Borghs, P. Van Dorpe, Angew. Chem. Int. Ed. 2009, 48, 9932-9935.

[5] a) A. Hartschuh, E.J. Sánchez, X.S. Xie, L. Novotny, Phys. Rev. Lett. 2003, 90, 095503; b) T. Ichimura, N. Hayazawa, M. Hashimoto, Y. Inouye, S. Kawata, Phys. Rev. Lett. 2004, 92, 220801.

[6] S. Nanda, M.J. Kim, K.S. Yeom, S. An, H. Ju, D.K. Yi, Trends Anal. Chem. 2016, 80, 125-131.

[7] W. Ren, R. Saito, L. Gao, F. Zheng, Z. Wu, B. Liu, M. Furukawa, J. Zhao, Z. Chen, H.-M. Cheng, Phys. Rev. B 2010, 81, 035412.

[8] A. Shiotari, T. Kumagai, M. Wolf, J. Phys. Chem. C 2014, 118, 1180611812.

[9] C. Li, P. Fan, A. Liang, Q. Liu, Z. Jiang, Microchim. Acta 2018, 185, 177

[10] W. Xu, N. Mao, J. Zhang, Small 2013, 9, 1206-1224.

[11] a) S. Ben-Valid, H. Dumortier, M. Decossas, R. Sfez, M. Meneghetti, A. Bianco, S. Yitzchaik, J. Mater. Chem. 2010, 20, 2408-2417; b) A. Nunes, C. Bussy, L. Gherardini, M. Meneghetti, M.A. Herrero, A. Bianco, M. Prato, T. Pizzorusso, K. Al-Jamal, K. Kostarelos, Nanomedicine 2012, 7, 1485-1494.

[12] a) Keren, C. Zavaleta, Z. Cheng, A. de la Zerda, O. Gheysens, S.S Gambhir, Proc. Natl. Acad. Sci. 2008, 105, 5844-5849; b) K. Cheng, Z. Cheng, Curr. Med. Chem. 2012, 19, 4767-4785.

[13] H. Wang, Y. Wang, Z. Hu, X. Wang, ACS Appl. Mater. Interfaces 2012 4, 6827-6834.

[14] D. Kosynkin, A. Higginbotham, A. Sinitskii, J. Lomeda, A. Dimiev, K. Price, J.M. Tour, Nature 2009, 468, 872-876.

[15] A. Dimiev, A. Khannanov, I. Vakhitov, A. Kiiamov, K. Shukhina, J.M. Tour, ACS Nano 2018, 12, 3985-3993.

[16] T.M. Swager, ACS Macro Lett. 2012, 1, 3-5.

[17] a) A.C. Dillon, M. Yudasaka, M.S. Dresselhaus, J. Nanosci. Nanotechnol. 2004, 4, 691-703; b) M.S. Dresselhaus, A. Jorio, M. Hofmann, G. Dresselhaus, R. Saito, Nano Lett. 2010, 10, 751-758.

[18] J. Casado, V. Hernández, J.T. López-Navarrete, Chem. Rec. 2015, 15, 1110-1118.

[19] a) U. Hohenester, A. Trügler, Comput. Phys. Commun. 2012, 183, 370; b) U. Hohenester, Comput. Phys. Commun. 2018, 222, 209-228.

[20] E. Pensa, E. Cortés, G. Corthey, P. Carro, C. Vericat, M.H. Fonticelli, G. Benítez, A.A. Rubert, R.C. Salvarezza, Acc. Chem. Res. 2012, 45, 1183-1192.

[21] Z. Lu, H. Si, Z. Li, J. Yu, Y. Liu, D. Feng, C. Zhang, W. Yang, B. Man, S. Jiang, Optics Express 2018, 26, 21626

[22] a) M. Quintana, A. Montellano, A.E. del Rio Castillo, G. Van Tendeloo, C. Bittencourt, M. Prato, Chem. Commun. 2011, 47, 9330-9332; b) B.V. Enüstün, J. Turkevich, J. Am. Chem. Soc. 1963, 85, 3317-3328.

[23] A. G. Skirtach, P. Karageorgiev, M. F. Bédard, G. B. Sukhorukov, H. Möhwald. J. Am. Chem. Soc. 2008, 130, 11572-11573.

[24] L. Beqa, A. Kumar, Z. Fan, D. Senapati, P.C. Ray, Chem. Phys. Lett. 2011, 512, 237-242.

[25] A.J. Caires, R.P. Vaz, C. Fantini, L.O. Ladeira, J. Colloid. Interf. Sci. 2015, 455, 78-82.

[26] a) J. Huang, L. Zhang, B. Chen, N. Ji, F. Chen, Y. Zhang, Z. Zhang, Nanoscale 2010, 2, 2733-2738; b) W.L. Fu, S.J. Zhen, C.Z. Huang, Analyst 2013, 138, 3075-3081.

[27] L. Qu, N. Wang, H. Xu, W. Wang, Y. Liu, L. Kuo, T. P. Yadav, J. Wu, J. Joyner, Y. Song, H. Li, J. Lou, R. Vajtai, P. M. Ajayan. Adv. Funct. Mater. 2017, 27, 1701714

[28] D. Wang, X. Zhao, Z. Gu. Optics Commun. 2017, 395, 3-15.

[29] E. Oh, K. Susumu, A.J. Mäkinen, J.R. Deschamps, A.L. Huston, I.L. Medintz, J. Phys. Chem. C 2013, 117, 18947-18956.

[30] A.L. Higginbotham, D.V. Kosynkin, A. Sinitskii, Z. Sun, J.M. Tour, ACS Nano 2010, 4, 2059-2069.

[31] D.A. Pearson, M. Blanchette, M.L. Baker, C.A. Guindon, Tetrahedron Lett. 1989, 30, 2739-2742. 
[32] V. Amendola, M. Meneghetti, J. Mater. Chem. 2007, 17, 4705-4710.

[33] C. Fabbro, T. Da Ros, Carbon 2014, 76, 96104.
[34] R. Esteban, A. G. Borisov, P. Nordlander, J. Aizpurua. Nature Commun. 2012, 3 , 825 


\section{Entry for the Table of Contents}

\section{FULL PAPER}

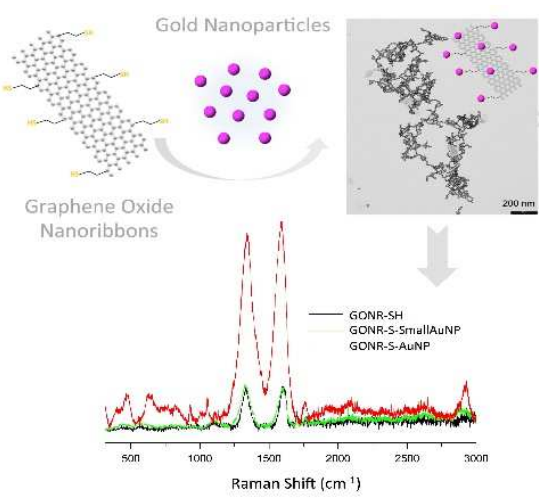

Jose M. González-Domínguez, * Andrea Colusso, Lucio Litti, Adrian Ostric,

Moreno Meneghetti, * Tatiana Da Ros *

Page No. - Page No.

Thiolated Graphene Oxide

Nanoribbons as templates for 2D SERS nanostructure.

Bond, Gold Bond. Functionalizing graphene oxide nanoribbons with terminal thiol groups greatly enhances the binding to gold nanoparticles, providing a SERS effect. A twodimensional aggregation pattern is the responsible of such effect, leading to robust hybrid nanomaterials. These hybrids are a promising platform for SERS sensing. 